\title{
ABOUT THE STEIN EQUATION FOR THE GENERALIZED INVERSE GAUSSIAN AND KUMMER DISTRIBUTIONS
}

\author{
Essomanda Konzou ${ }^{1,2}$ And Angelo Efoevi Koudou ${ }^{1, *}$
}

\begin{abstract}
We observe that the density of the Kummer distribution satisfies a certain differential equation, leading to a Stein characterization of this distribution and to a solution of the related Stein equation. A bound is derived for the solution and for its first and second derivatives. To provide a bound for the solution we partly use the same framework as in Gaunt 2017 [Stein, ESAIM: PS 21 (2017) 303$316]$ in the case of the generalized inverse Gaussian distribution, which we revisit by correcting a minor error. We also bound the first and second derivatives of the Stein equation in the latter case.
\end{abstract}

Mathematics Subject Classification. 60F05, 60E05.

Received August 2, 2018. Accepted February 5, 2020.

\section{INTRODUCTION}

For $a>0, b \in \mathbb{R}, c>0$, the Kummer distribution with parameters $a, b, c$ has density

$$
k_{a, b, c}(x)=\frac{1}{\Gamma(a) \psi(a, a-b+1 ; c)} x^{a-1}(1+x)^{-a-b} e^{-c x},(x>0)
$$

where $\psi$ is the confluent hypergeometric function of the second kind.

The generalized inverse Gaussian (hereafter $G I G$ ) distribution with parameters $p \in \mathbb{R}, a>0, b>0$ has density

$$
g_{p, a, b}(x)=\frac{(a / b)^{p / 2}}{2 K_{p}(\sqrt{a b})} x^{p-1} e^{-\frac{1}{2}(a x+b / x)}, \quad x>0,
$$

where $K_{p}$ is the modified Bessel function of the third kind.

For details on GIG and Kummer distributions see [6, 7, 10] and references therein, where one can see for instance that these distributions are involved in some characterization problems related to the so-called Matsumoto-Yor property.

In this paper, these two distributions are considered in the context of Stein's method. This method introduced in [14] is a technique used to bound the error in the approximation of the distribution of a random variable of

Keywords and phrases: Generalized inverse Gaussian distribution, Kummer distribution, Stein characterization.

1 Université de Lorraine, CNRS, IECL, 54000 Nancy, France.

2 Université de Lomé, Lomé, Togo.

* Corresponding author: efoevi.koudou@univ-lorraine.fr 
interest by another probability (for instance the normal) distribution. For an overview of Stein's method see $[1,11]$. The first steps of this method consist in finding an operator called Stein operator characterizing the targeted distribution, then solving the corresponding so-called Stein equation.

One finds in [14] a seminal instance of the method, where Stein showed that a random variable $X$ has a standard normal distribution if and only if for all real-valued absolutely continuous function $f$ such that $\mathbb{E}\left|f^{\prime}(Z)\right|<\infty$ for $Z \sim N(0,1)$

$$
\mathbb{E}\left[f^{\prime}(X)-X f(X)\right]=0
$$

The corresponding Stein equation is

$$
f^{\prime}(x)-x f(x)=h(x)-\mathbb{E} h(Z)
$$

where $h$ is a bounded function and $Z$ a random variable following the standard normal distribution. The operator $f \mapsto T_{f}$ defined by $\left(T_{f}\right)(x)=f^{\prime}(x)-x f(x)$ is the corresponding Stein operator.

If a function $f_{h}$ is a solution of the previous equation, then for any random variable $U$ we have

$$
\left|\mathbb{E}\left(f_{h}^{\prime}(U)-U f_{h}(U)\right)\right|=|\mathbb{E}(h(U))-\mathbb{E}(h(Z))| .
$$

Thus, in order to bound $|\mathbb{E}(h(U))-\mathbb{E}(h(Z))|$ given $h$, its enough to find a solution $f_{h}$ of the Stein equation and to bound the left-hand side of the previous equation. The problem of solving the Stein equation for other distributions than the standard normal distribution and bounding the solution and its derivatives has been widely studied in the literature (see [4] among many others).

The aim of this paper is to solve the Stein equation and derive a bound of the solution and of its first and second derivatives for the Kummer distribution and for the generalized inverse Gaussian distribution. The idea of this paper emerged by reading the remarkable work by [2] about a Stein characterization of the generalized hyperbolic distribution of which the generalized inverse Gaussian distribution (GIG) is a limiting case. Among many other results, [2] solved the GIG Stein equation and bounded the solution by using a general result obtained in [12] when the targeted distribution has a density $g$ satisfying

$$
(s(x) g(x))^{\prime}=\tau(x) g(x)
$$

for some polynomial functions $s$ and $\tau$. Also a bound was obtained for the solution under the condition that the function $\tau$ be a decreasing linear function. But since this linearity condition does not hold in the GIG case, the bound given by [2] has to be slightly corrected. This is done in Theorem 3.2 after recalling the general framework of Schoutens [12] and adapting it to the cases where $\tau$ is decreasing but not necessarily linear. Indeed, we realized that the procedure adopted in [12] still works, via a slight change, even if $\tau$ is not linear.

Observing that the Kummer density also satisfies (1.1), we can use the same methodology (Thm. 4.2) for this distribution. We begin by putting the restrictions $p \leq-1$ for the GIG density and $1-b-c \leq 0$ for the Kummer density in order for the corresponding function $\tau$ to be decreasing on $(0, \infty)$. But we finally derived bounds without these restrictions, due to the form of the function $\tau$ in the GIG and Kummer cases.

In Section 2 we recall the general framework established by [12] for densities $g$ satisfying (1.1) without the assumption of linearity of $\tau$, but assuming that $\tau$ is decreasing. We retrieve the Stein operator given in [12] by using the density approach initiated in [15] and further developed in [9]. In the same section we bound the solution in a setting where the function $\tau$ is not decreasing but has a particular behaviour shared by the GIG and Kummer cases. The results are applied to the GIG distribution in Section 3 (the proof of the bound of the second derivative is postponed to the Appendix A), and to the Kummer one in Section 4. In Section 5 we give some examples of possible applications that will be investigated in future work. 


\section{Stein characterization, solution of the Stein Equation and BOUND FOR THE SOLUTION IN THE SCHOUTENS FRAMEWORK}

Theorem 1 in [12] addressed the problem of establishing a Stein characterization for probability distributions with density $g$ satisfying (1.1) for some polynomial functions $s$ et $\tau$, and proved that a Stein operator in this case is $f \mapsto s f^{\prime}+\tau f$. We realized (see the following theorem) that the same Stein operator can be arrived at by using the density approach designed in [15] and [9]. The support of the density may be any interval, but here we take this support to be $(0, \infty)$ in the purpose of the application to the GIG and Kummer distributions.

Theorem 2.1. Consider a density $g$ on $(0, \infty)$ such that (1.1) holds for some polynomial functions $s$ and $\tau$. Then a positive random variable $X$ has density $g$ if and only if for any differentiable function $f$ such that $\lim _{x \rightarrow 0} s(x) g(x) f(x)=\lim _{x \rightarrow \infty} s(x) g(x) f(x)=0$,

$$
\mathbb{E}\left[s(X) f^{\prime}(X)+\tau(X) f(X)\right]=0 .
$$

Proof. We use Corollary 2.1 of [9]. According to this corollary, a Stein operator related to the density function $g$ is

$$
T_{g} f(x)=\frac{1}{g(x)}(f g)^{\prime}(x)
$$

Applyng this operator to $s f$, we have

$$
\begin{aligned}
T_{g}(s f)(x) & =\frac{1}{g(x)}(s f g)^{\prime}(x) \\
& =\frac{1}{g(x)}\left(f^{\prime}(x) s(x) g(x)+f(x)(s g)^{\prime}(x)\right)
\end{aligned}
$$

which, by (1.1), reads

$$
\begin{aligned}
T_{g}(s f)(x) & =\frac{1}{g(x)}\left(f^{\prime}(x) s(x) g(x)+f(x) \tau(x) g(x)\right) \\
& =f^{\prime}(x) s(x)+f(x) \tau(x) .
\end{aligned}
$$

Theorem 2.1 shows that the Stein equation related to any density $g$ satisfying (1.1) enjoys the tractable form

$$
s(x) f^{\prime}(x)+\tau(x) f(x)=h(x)-\mathbb{E} h(W)
$$

where $W$ is random variable with density $g$. Schoutens [12] found a solution to the Stein equation (2.1) and established a bound for the solution, under the condition that the function $\tau$ be a decreasing linear function (which is the case for the so-called Pearson and Ord classes of distributions considered in [12]).

The following result comes from Proposition 1 in [12]. We again take the support of the density function to be $(0, \infty)$. 
Proposition 2.2. Consider a density function $g>0$ on $(0, \infty)$ satisfying equation (1.1), for some polynomial functions $s$ and $\tau$. Then a solution of the Stein equation (2.1) is

$$
\begin{aligned}
f_{h}(x) & =\frac{1}{s(x) g(x)} \int_{0}^{x} g(t)[h(t)-\mathbb{E} h(W)] \mathrm{d} t \\
& =\frac{-1}{s(x) g(x)} \int_{x}^{+\infty} g(t)[h(t)-\mathbb{E} h(W)] \mathrm{d} t .
\end{aligned}
$$

Remark 2.3. The proof of this proposition is given in [12] just by calculating the derivative of the function $f_{h}$ defined by (2.2) and checking directly that $f_{h}$ satisfies (2.1). Our following proposition complements this result.

Proposition 2.4. Under the notation and assumptions of Proposition 2.2,

- The solutions of the Stein equation (2.1) are of the form

$$
\begin{aligned}
f_{h}(x) & =\frac{1}{s(x) g(x)} \int_{0}^{x} g(t)[h(t)-\mathbb{E} h(W)] \mathrm{d} t+\frac{C}{s(x) g(x)} \\
& =\frac{-1}{s(x) g(x)} \int_{x}^{+\infty} g(t)[h(t)-\mathbb{E} h(W)] \mathrm{d} t+\frac{C}{s(x) g(x)}
\end{aligned}
$$

where $C$ is constant.

- Suppose $\lim _{x \rightarrow 0} s(x) g(x)=0$. For the solution to be bounded, it is necessary that $C=0$ in $(2.3)$.

Proof. Multiplying both sides of (2.1) by $g(x)$ we have

$$
s(x) g(x) f^{\prime}(x)+\tau(x) g(x) f(x)=g(x)(h(x)-\mathbb{E} h(W))
$$

which, by (1.1), can be written

$$
s(x) g(x) f^{\prime}(x)+(s g)^{\prime}(x) f(x)=g(x)(h(x)-\mathbb{E} h(W)),
$$

i.e.

$$
(s g f)^{\prime}(x)=g(x)(h(x)-\mathbb{E} h(W)) .
$$

As a consequence, there exists a constant $C$ such that

$$
s(x) g(x) f(x)=\int_{0}^{x} g(t)[h(t)-\mathbb{E} h(W)] \mathrm{d} t+C
$$

which implies (2.3).

Suppose $f$ is bounded. Since $\lim _{x \rightarrow 0} s(x) g(x)=0$, letting $x$ tend to 0 in (2.4) yields $C=0$.

The second expression for $f_{h}$ follows from the fact that, since $W$ has density $g$,

$$
\int_{0}^{+\infty} g(t)[h(t)-\mathbb{E} h(W)] \mathrm{d} t=0
$$


The following proposition proves that the solution given by (2.2) is bounded indeed if $h$ is bounded, and thus is the unique bounded solution to the Stein equation associated to the density $g$. A bound of this solution is provided.

Proposition 2.5. Consider a density function $g>0$ on $(0, \infty)$ satisfying equation (1.1), where $s$ and $\tau$ are polynomial functions such that $s>0$ on $(0, \infty)$. Assume that $\lim _{x \rightarrow 0} s(x) g(x)=\lim _{x \rightarrow \infty} s(x) g(x)=0$.

If $h$ is a bounded continuous function, then

$$
\left\|f_{h}\right\| \leq M\|h(\cdot)-\mathbb{E} h(W)\|
$$

where $W$ has density $g, \| f_{h}||=\sup _{x>0}\left|f_{h}(x)\right|$ and the constant $M$ is defined as follows:

1. Suppose $\tau$ is decreasing and has a unique zero $\alpha$ on $(0, \infty)$. Then

$$
M=\max \left(\frac{1}{s(\alpha) g(\alpha)} \int_{0}^{\alpha} g(t) \mathrm{d} t ; \frac{1}{s(\alpha) g(\alpha)} \int_{\alpha}^{+\infty} g(t) \mathrm{d} t\right) .
$$

2. Assume that $\tau(0)>0$ and, for some $\beta>0, \tau$ is increasing on $(0, \beta]$, decreasing on $[\beta, \infty)$ and has a unique zero $\alpha$ on $[\beta, \infty)$. Then

$$
M=\max \left(\frac{1}{\tau(0)} ; \frac{1}{s(\beta) g(\beta)}\right)
$$

Remark 2.6. The first item of Proposition 2.5 is a reformulation of Lemma 1 in [12] without the assumption that $\tau$ is linear. With this assumption, [12] established the same bound with $\alpha=\mathbb{E}(W)$ (for a random variable $W$ with density $g$ ), which is not true if $\tau$ is not linear. The proof given below follows the lines of that of [12] where we observed that the assumption of linearity of $\tau$ was used nowhere except to state that its only zero is $\alpha=\mathbb{E}(W)$.

The proof of item 1 of Proposition 2.5 uses the following lemma:

Lemma 2.7. Under the assumptions of Proposition 2.5,

$$
\int_{0}^{x} g(t) \mathrm{d} t \leq \frac{s(x) g(x)}{\tau(x)} \quad \text { for } \quad x<\alpha
$$

and

$$
\int_{x}^{+\infty} g(t) \mathrm{d} t \leq \frac{-s(x) g(x)}{\tau(x)} \quad \text { for } \quad x>\alpha .
$$


Proof. Suppose $x<\alpha$. Since $\tau$ is positive and decreasing on $(0, \alpha)$, we have $\frac{\tau(t)}{\tau(x)} \geq 1$ for all $t \leq x$. Therefore

$$
\begin{aligned}
\int_{0}^{x} g(t) \mathrm{d} t & \leq \int_{0}^{x} g(t) \frac{\tau(t)}{\tau(x)} \mathrm{d} t \\
& =\frac{1}{\tau(x)} \int_{0}^{x} \tau(t) g(t) \mathrm{d} t \\
& =\frac{s(x) g(x)}{\tau(x)}
\end{aligned}
$$

because of (1.1) and as $\lim _{t \rightarrow 0} s(t) g(t)=0$.

For $x>\alpha$, since $\tau$ is negative and decreasing on $(\alpha, \infty)$, we have $\frac{\tau(t)}{\tau(x)} \geq 1$ for all $t \geq x$. As a consequence,

$$
\begin{aligned}
\int_{x}^{+\infty} g(t) \mathrm{d} t & \leq \int_{x}^{+\infty} g(t) \frac{\tau(t)}{\tau(x)} \mathrm{d} t \\
& =\frac{-s(x) g(x)}{\tau(x)}
\end{aligned}
$$

since $\lim _{t \rightarrow \infty} s(t) g(t)=0$.

Now, let us prove the item 1 of Proposition 2.5.

Proof. For $x<\alpha$,

$$
\begin{aligned}
\left|f_{h}(x)\right| & =\left|\frac{1}{s(x) g(x)} \int_{0}^{x} g(t)[h(t)-\mathbb{E} h(W)] \mathrm{d} t\right| \\
& \leq \frac{1}{s(x) g(x)} \int_{0}^{x} g(t)|h(t)-\mathbb{E} h(W)| \mathrm{d} t \\
& \leq\|h(\cdot)-\mathbb{E} h(W)\| \frac{1}{s(x) g(x)} \int_{0}^{x} g(t) \mathrm{d} t .
\end{aligned}
$$

Let $l(x)=\frac{1}{s(x) g(x)} \int_{0}^{x} g(t) \mathrm{d} t$. Then $l$ is differentiable on $(0, \infty)$ and

$$
\begin{aligned}
l^{\prime}(x) & =\frac{-(s(x) g(x))^{\prime}}{(s(x) g(x))^{2}} \int_{0}^{x} g(t) \mathrm{d} t+\frac{1}{s(x)} \\
& =\frac{-\tau(x)}{s^{2}(x) g(x)} \int_{0}^{x} g(t) \mathrm{d} t+\frac{1}{s(x)} .
\end{aligned}
$$

Using Lemma 2.7, we conclude that $l^{\prime}(x) \geq 0$. Then $l(x) \leq l(\alpha)$.

For $x>\alpha$,

$$
\left|f_{h}(x)\right| \leq\|h(x)-\mathbb{E} h(W)\| \frac{1}{s(x) g(x)} \int_{x}^{+\infty} g(t) \mathrm{d} t
$$


Let $u(x)=\frac{1}{s(x) g(x)} \int_{x}^{+\infty} g(t) \mathrm{d} t$. The function $u$ is differentiable on $(0, \infty)$ and

$$
u^{\prime}(x)=\frac{-\tau(x)}{s^{2}(x) g(x)} \int_{x}^{+\infty} g(t) \mathrm{d} t-\frac{1}{s(x)} .
$$

By Lemma 2.7, we conclude that $u^{\prime}(x) \leq 0$. Then $u(x) \leq u(\alpha)$.

Proof of item 2 of Proposition 2.5.

$\tau$ is increasing and positive on $[0, \beta]$, so that $\frac{\tau(t)}{\tau(0)} \geq 1$ for $0 \leq t \leq \beta$. Thus, for $x \leq \beta$,

$$
\begin{aligned}
\left|f_{h}(x)\right| & =\left|\frac{1}{s(x) g(x)} \int_{0}^{x} g(t)[h(t)-\mathbb{E} h(W)] \mathrm{d} t\right| \\
& \leq \frac{1}{s(x) g(x)} \int_{0}^{x} g(t)|h(t)-\mathbb{E} h(W)| \mathrm{d} t \\
& \leq\|h(\cdot)-\mathbb{E} h(W)\| \frac{1}{s(x) g(x)} \int_{0}^{x} g(t) \mathrm{d} t \\
& \leq\|h(\cdot)-\mathbb{E} h(W)\| \frac{1}{s(x) g(x)} \int_{0}^{x} \frac{\tau(t)}{\tau(0)} g(t) \mathrm{d} t \\
& =\|h(\cdot)-\mathbb{E} h(W)\| \frac{1}{\tau(0) s(x) g(x)} \int_{0}^{x}(s(t) g(t))^{\prime} \mathrm{d} t \\
& =\frac{1}{\tau(0)}\|h(\cdot)-\mathbb{E} h(W)\| .
\end{aligned}
$$

For $\beta \leq x \leq \alpha$, we have again

$$
\begin{aligned}
\left|f_{h}(x)\right| & \leq\|h(\cdot)-\mathbb{E} h(W)\| \frac{1}{s(x) g(x)} \int_{0}^{x} g(t) \mathrm{d} t \\
& \leq\|h(\cdot)-\mathbb{E} h(W)\| \frac{1}{s(x) g(x)} \\
& \leq\|h(\cdot)-\mathbb{E} h(W)\| \frac{1}{s(\beta) g(\beta)}
\end{aligned}
$$

because $x \mapsto s(x) g(x)$ is increasing on $[\beta, \alpha]$ (its derivative is $\tau(x) g(x)$ which is positive on $[\beta, \alpha]$ ).

For $x>\alpha$, an argument similar to the one used in the proof of Proposition 2.5 yields

$$
\begin{aligned}
\left|f_{h}(x)\right| & \leq\|h(\cdot)-\mathbb{E} h(W)\| \frac{1}{s(\alpha) g(\alpha)} \int_{x}^{+\infty} g(t) \mathrm{d} t \\
& \leq\|h(\cdot)-\mathbb{E} h(W)\| \frac{1}{s(\alpha) g(\alpha)} \\
& \leq\|h(\cdot)-\mathbb{E} h(W)\| \frac{1}{s(\beta) g(\beta)} .
\end{aligned}
$$


Remark 2.8. Note that [13] obtained bounds for the solution of the Stein equation and its first derivative in another general context where $\tau$ is not decreasing, under other assumptions that are not needed in the GIG and Kummer cases, where we obtain more explicit bounds, as shown in the two next sections.

\section{About the Stein equation of the Generalized inverse GAUSSIAN DISTRIBUTION}

Recall that the density of the GIG distribution with parameters $p \in \mathbb{R}, a>0, b>0$ is

$$
g_{p, a, b}(x)=\frac{(a / b)^{p / 2}}{2 K_{p}(\sqrt{a b})} x^{p-1} e^{-\frac{1}{2}(a x+b / x)}, \quad x>0,
$$

where $K_{p}$ is the modified Bessel function of the third kind.

Let

$$
s(x)=x^{2} \quad \text { and } \quad \tau_{p, a, b}(x)=\frac{b}{2}+(p+1) x-\frac{a}{2} x^{2} .
$$

Then, as observed by [2], the GIG density $g_{p, a, b}$ satisfies

$$
\left(s(x) g_{p, a, b}(x)\right)^{\prime}=\tau_{p, a, b}(x) g_{p, a, b}(x) .
$$

This enables us to apply Theorem 2.1 to retrieve the following Stein characterization of the GIG distribution given in [7] and [2]:

Proposition 3.1. A random variable $X$ follows the $G I G$ distribution with density $g_{p, a, b}$ if and only if, for all real-valued and differentiable function $f$ such that $\lim _{x \rightarrow \infty} g_{p, a, b}(x) f(x)=\lim _{x \rightarrow 0} g_{p, a, b}(x) f(x)=0$, and such that the following expectation exists, we have:

$$
\mathbb{E}\left[X^{2} f^{\prime}(X)+\left(\frac{b}{2}+(p+1) X-\frac{a}{2} X^{2}\right) f(X)\right]=0 .
$$

The corresponding Stein equation is

$$
x^{2} f^{\prime}(x)+\left(\frac{b}{2}+(p+1) x-\frac{a}{2} x^{2}\right) f(x)=h(x)-\mathbb{E} h(W)
$$

where $h$ is a bounded function and $W$ a random variable following the $G I G$ distribution with parameters $p, a, b$.

\subsection{Bound of the solution for $p \leq-1$}

We apply Propositions 2.4 and 2.5 to solve equation (3.4) and bound the solution. Let us check that the assumptions of these propositions are true in the GIG case.

Firstly, we note that, by (3.1),

$$
s(x) g_{p, a, b}(x)=\frac{(a / b)^{p / 2}}{2 K_{p}(\sqrt{a b})} x^{p+1} e^{-\frac{1}{2}(a x+b / x)}, \quad x>0,
$$

which shows that $\lim _{x \rightarrow \infty} s(x) g_{p, a, b}(x)=\lim _{x \rightarrow 0} s(x) g_{p, a, b}(x)=0$. 
Secondly, observe that if $p \leq-1$, then the function $\tau_{p, a, b}$ defined by $(3.2)$ is decreasing on $(0, \infty)$ and that its only zero on $(0, \infty)$ is $\alpha=\frac{p+1+\sqrt{(p+1)^{2}+a b}}{a}$.

Thus, by using Propositions 2.4 and 2.5 , we obtain the following theorem.

Theorem 3.2. The GIG Stein equation (3.4) has solution

$$
\begin{aligned}
f_{h}(x) & =\frac{1}{s(x) g_{p, a, b}(x)} \int_{0}^{x} g_{p, a, b}(t)[h(t)-\mathbb{E} h(W)] \mathrm{d} t \\
& =\frac{-1}{s(x) g_{p, a, b}(x)} \int_{x}^{+\infty} g_{p, a, b}(t)[h(t)-\mathbb{E} h(W)] \mathrm{d} t
\end{aligned}
$$

where $W$ follows the GIG distribution with parameters $p, a, b$.

If $h$ is a bounded continuous function and $p \leq-1$, then the function defined by (3.5) is the unique bounded solution of (3.4) and

$$
\left\|f_{h}\right\| \leq M\|h(\cdot)-\mathbb{E} h(W)\|
$$

where

$$
\begin{gathered}
\alpha=\frac{p+1+\sqrt{(p+1)^{2}+a b}}{a} \\
M=\max \left(\frac{1}{\alpha^{2} g_{p, a, b}(\alpha)} \int_{0}^{\alpha} g_{p, a, b}(t) \mathrm{d} t ; \frac{1}{\alpha^{2} g_{p, a, b}(\alpha)} \int_{\alpha}^{+\infty} g_{p, a, b}(t) \mathrm{d} t\right) .
\end{gathered}
$$

Remark 3.3. This result was claimed by Gaunt (see [2]) with $\alpha=\mathbb{E}(W)$ by applying Proposition 1 of [12]. The only slight mistake is that $\tau_{p, a, b}$ is not a polynomial function of degree one as in [12].

Remark 3.4. By bounding by 1 the integrals in equation (3.7), one obtains

$$
\begin{aligned}
\left\|f_{h}\right\| & \leq \frac{1}{\alpha^{2} g_{p, a, b}(\alpha)}\|h(\cdot)-\mathbb{E} h(W)\| \\
& =\frac{a^{2}}{\left(a b+2(p+1)\left(p+1+\sqrt{(p+1)^{2}+a b}\right)\right) g_{p, a, b}\left(\frac{p+1+\sqrt{(p+1)^{2}+a b}}{a}\right)}\|h(\cdot)-\mathbb{E} h(W)\| .
\end{aligned}
$$

\subsection{Bound of the solution for $p>-1$}

Theorem 3.5. Consider $p>-1, a>0, b>0$ and $\alpha$ defined by (3.6). Let $h:(0,+\infty) \rightarrow \mathbb{R}$ be a continuous bounded function. The solution $f_{h}$ given by (3.5) of the Stein equation for the $G I G(p, a, b)$ distribution with density $g_{p, a, b}$ is such that

$$
\left\|f_{h}\right\| \leq \max \left(\frac{2}{b}, \frac{a^{2}}{(p+1)^{2} g_{p, a, b}\left(\frac{p+1}{a}\right)}\right)\|h(\cdot)-\mathbb{E} h(W)\| .
$$

Proof. We use the item 2 of Proposition 2.5 with $\tau(x)=\tau_{p, a, b}(x)=\frac{b}{2}+(p+1) x-\frac{a}{2} x^{2}, \tau(0)=b / 2$ and $\beta=$ $(p+1) / a$. 


\subsection{Bound of the first derivative}

Theorem 3.6. Let $W \sim G I G(p, a, b)$. Consider again $\alpha$ defined by (3.6), let

$$
\alpha_{1}=\frac{p+3+\sqrt{(p+3)^{2}+a b}}{a}
$$

and let $M$ be given by

$$
M=\left\{\begin{array}{l}
\max \left(\frac{1}{\alpha^{2} g_{p, a, b}(\alpha)} \int_{0}^{\alpha} g_{p, a, b}(t) \mathrm{d} t ; \frac{1}{\alpha^{2} g_{p, a, b}(\alpha)} \int_{\alpha}^{+\infty} g_{p, a, b}(t) \mathrm{d} t\right) \quad \text { if } p \leq-1 \\
\max \left(\frac{2}{b}, \frac{a^{2}}{(p+1)^{2} g_{p, a, b}\left(\frac{p+1}{a}\right)}\right) \quad \text { if } p>-1 .
\end{array}\right.
$$

Let $h:(0,+\infty) \rightarrow \mathbb{R}$ be a bounded, differentiable function with bounded derivative $h^{\prime}$. Consider

$$
\begin{gathered}
C_{1}=\frac{2}{b}\left(\left\|h^{\prime}\right\|+\max (2,|p+1|) M\|h(\cdot)-\mathbb{E} h(W)\|\right), \\
C_{2}=\left(\left\|h^{\prime}\right\|+\left(2+\sqrt{(p+3)^{2}+a b}\right) M\|h(\cdot)-\mathbb{E} h(W)\|\right) \frac{1}{\alpha_{1}^{2} g_{p+2, a, b}\left(\alpha_{1}\right)} \int_{0}^{\alpha_{1}} g_{p+2, a, b}(t) \mathrm{d} t, \\
C_{3}=\frac{2}{\alpha_{1}^{2}}\|h(\cdot)-\mathbb{E} h(W)\|, \\
C_{4}=\left(\left\|h^{\prime}\right\|+\left(2+\sqrt{(p+3)^{2}+a b}\right) M\|h(\cdot)-\mathbb{E} h(W)\|\right) \frac{a^{2}}{(p+3)^{2} g_{p+2, a, b}\left(\frac{p+3}{a}\right)} .
\end{gathered}
$$

The derivative $f_{h}^{\prime}$ of the solution $f_{h}$ of equation (3.4) is such that

$$
\left\|f_{h}^{\prime}\right\| \leq M^{\prime}=\left\{\begin{array}{l}
\max \left(C_{2}, C_{3}\right) \text { if } p \leq-3 \\
\max \left(C_{1}, C_{3}, C_{4}\right) \text { if } p>-3 .
\end{array}\right.
$$

Proof. First, assume that $p \leq-3$.

$f_{h}$ satisfies the differential equation

$$
x^{2} f^{\prime \prime}(x)+\left(\frac{b}{2}+(p+3) x-\frac{a}{2} x^{2}\right) f^{\prime}(x)=h^{\prime}(x)-(p+1-a x) f(x) .
$$

Let $h_{1}(x)=h^{\prime}(x)-(p+1-a x) f_{h}(x)$. Then, by equation $(3.11), f_{h}^{\prime}$ solves the differential equation

$$
x^{2} f^{\prime}(x)+\left(\frac{b}{2}+(p+3) x-\frac{a}{2} x^{2}\right) f(x)=h_{1}(x) .
$$


Since $\mathbb{E} h_{1}(Z)=\mathbb{E}\left(Z^{2} f^{\prime}(Z)+\left(\frac{b}{2}+(p+3) Z-\frac{a}{2} Z^{2}\right) f(Z)=0\right.$ if $Z \sim G I G(p+2, a, b)$ by the Stein characterization of $G I G(p+2, a, b)$, then equation (3.12) is the $G I G(p+2, a, b)$ Stein equation. An instance of such a strategy can be found in [3] when bounding the derivatives of the Gamma Stein equation.

Thus, for $p \leq-3$, i.e. $p+2 \leq-1$, we can apply the same argument as in Theorem 3.2 to state that if $x<\alpha_{1}$ and $h_{1}$ is bounded, then

$$
\left|f_{h}^{\prime}(x)\right| \leq\left\|h_{1}\right\| \frac{1}{s\left(\alpha_{1}\right) g_{1}\left(\alpha_{1}\right)} \int_{0}^{\alpha_{1}} g_{1}(t) \mathrm{d} t
$$

where $g_{1}:=g_{p+2, a, b}$ is the density of the $G I G(p+2, a, b)$ distribution and $\alpha_{1}=\frac{p+3+\sqrt{(p+3)^{2}+a b}}{a}$.

We have

$$
\begin{aligned}
\left|h_{1}(x)\right| & =\left|h^{\prime}(x)-(p+1-a x) f(x)\right| \\
& \leq\left\|h^{\prime}\right\|+|p+1-a x|\left\|f_{h}\right\| \\
& =\left\|h^{\prime}\right\|+(-p-1+a x)\left\|f_{h}\right\| \quad(p+1-a x<0 \quad \forall p \leq-3, x>0) \\
& \leq\left\|h^{\prime}\right\|+\left(-p-1+a \alpha_{1}\right)\left\|f_{h}\right\| \quad\left(x<\alpha_{1}\right) \\
& =\left\|h^{\prime}\right\|+\left(2+\sqrt{(p+3)^{2}+a b}\right)\left\|f_{h}\right\| \\
& \leq\left\|h^{\prime}\right\|+\left(2+\sqrt{(p+3)^{2}+a b}\right) M\|h(\cdot)-\mathbb{E} h(W)\| .
\end{aligned}
$$

Hence, for $p \leq-3$ and $x \leq \alpha_{1}$,

$$
\left|f_{h}^{\prime}(x)\right| \leq\left(\left\|h^{\prime}\right\|+\left(2+\sqrt{(p+3)^{2}+a b}\right) M\|h(\cdot)-\mathbb{E} h(W)\|\right) \frac{1}{s\left(\alpha_{1}\right) g_{1}\left(\alpha_{1}\right)} \int_{0}^{\alpha_{1}} g_{1}(t) \mathrm{d} t .
$$

If $p \leq-3$ and $x \geq \alpha_{1}$, the function $\tau:=\tau_{p, a, b}$ (given by (3.2)) is decreasing and negative. By (3.5) and (3.3),

$$
\begin{gathered}
f_{h}^{\prime}(x)=\frac{h(x)-\mathbb{E} h(W)}{s(x)}-\frac{\tau(x) f_{h}(x)}{s(x)} \\
\left|f_{h}^{\prime}(x)\right| \leq \frac{\|h(\cdot)-\mathbb{E} h(W)\|}{s(x)}+\frac{\left|\tau(x) f_{h}(x)\right|}{s(x)} .
\end{gathered}
$$

Furthermore,

$$
\begin{aligned}
\left|\tau(x) f_{h}(x)\right| & =\left|\frac{-\tau(x)}{s(x) g(x)} \int_{x}^{+\infty} g(t)[h(t)-\mathbb{E} h(W)] \mathrm{d} t\right| \\
& \leq\|h(\cdot)-\mathbb{E} h(W)\| \frac{-\tau(x)}{s(x) g(x)} \int_{x}^{+\infty} g(t) \mathrm{d} t \quad(\tau \quad \text { is negative }) \\
& \leq\|h(\cdot)-\mathbb{E} h(W)\| \frac{-\tau(x)}{s(x) g(x)} \int_{x}^{+\infty} \frac{\tau(t)}{\tau(x)} g(t) \mathrm{d} t \quad\left(\frac{\tau(t)}{\tau(x)} \geq 1 \quad \forall x \leq t\right) \\
& =\|h(\cdot)-\mathbb{E} h(W)\| .
\end{aligned}
$$

Hence $\left|f_{h}^{\prime}(x)\right| \leq \frac{2}{s(x)}\|h(\cdot)-\mathbb{E} h(W)\| \leq \frac{2}{\alpha_{1}^{2}}\|h(\cdot)-\mathbb{E} h(W)\|$.

Now assume that $p>-3$. 
On $\left(0, \frac{p+3}{a}\right), \tau_{1}:=\tau_{p+2, a, b}$ is such that $\frac{\tau_{1}(t)}{\tau_{1}(0)} \geq 1 \forall 0 \leq t \leq \frac{p+3}{a}$ so that

$$
\begin{aligned}
\left|h_{1}(x)\right| & \leq\left\|h^{\prime}\right\|+|p+1-a x|\left\|f_{h}\right\| \\
& \leq\left\|h^{\prime}\right\|+\max (2,|p+1|)\left\|f_{h}\right\| \\
& \leq\left\|h^{\prime}\right\|+\max (2,|p+1|) M\|h(\cdot)-\mathbb{E} h(W)\|, \\
\left|f_{h}^{\prime}(x)\right| & \leq\left\|h_{1}\right\| \frac{1}{s(x) g_{1}(x)} \int_{0}^{x} g_{1}(t) \mathrm{d} t \\
& \leq\left\|h_{1}\right\| \frac{1}{\tau_{1}(0) s(x) g_{1}(x)} \int_{0}^{x} \tau_{1}(t) g_{1}(t) \mathrm{d} t \\
& =\frac{1}{\tau_{1}(0)}\left\|h_{1}\right\| \\
& =\frac{2}{b}\left\|h_{1}\right\|
\end{aligned}
$$

and

$$
\left\|f_{h}^{\prime}\right\| \leq \frac{2}{b}\left(\left\|h^{\prime}\right\|+\max (2,|p+1|) M\|h(\cdot)-\mathbb{E} h(W)\|\right) .
$$

On $\left(\frac{p+3}{a}, \infty\right)$, the function $\tau_{1}$ is decreasing and we use the same arguments as in the case $p \leq-3$.

\subsection{Bound of the second derivative}

Theorem 3.7. Let $W \sim G I G(p, a, b)$. Define $\alpha_{2}=\frac{p+5+\sqrt{(p+5)^{2}+a b}}{a}$ and denote by $g_{2}:=g_{p+4, a, b}$ the density of the $G I G(p+4, a, b)$ distribution.

Let $h:(0, \infty) \rightarrow \mathbb{R}$ be a bounded, twice differentiable function such that $h^{\prime}$ and $h^{\prime \prime}$ are bounded. The second derivative $f_{h}^{\prime \prime}$ of the solution $f_{h}$ of equation (3.4) is such that

$$
\left\|f_{h}^{\prime \prime}\right\| \leq M^{\prime \prime}=\left\{\begin{array}{l}
\max \left(C_{5}, C_{6}\right) \text { if } p \leq-5 \\
\max \left(C_{4}, C_{6}, C_{7}\right) \text { if } p>-5
\end{array}\right.
$$

where

$$
\begin{gathered}
C_{4}=\frac{2}{b}\left(\left\|h^{\prime \prime}\right\|+\max (6,|2 p+4|) M^{\prime}+a M\|h(\cdot)-\mathbb{E} h(W)\|\right), \\
C_{5}=\left(\left\|h^{\prime \prime}\right\|+\left(6+2 \sqrt{(p+5)^{2}+a b}\right) M^{\prime}+a M\|h(\cdot)-\mathbb{E} h(W)\|\right) \frac{1}{\alpha_{2}^{2} g_{2}\left(\alpha_{2}\right)} \int_{0}^{\alpha_{2}} g_{2}(t) \mathrm{d} t, \\
C_{6}=\frac{2}{\alpha_{2}^{2}}\left(\left\|h^{\prime}\right\|+\frac{4 a+a \sqrt{(p+5)^{2}+a b}}{4 p+20+4 \sqrt{(p+5)^{2}+a b}}\|h(\cdot)-\mathbb{E} h(W)\|\right),
\end{gathered}
$$




$$
C_{7}=\left(\left\|h^{\prime \prime}\right\|+\left(6+2 \sqrt{(p+5)^{2}+a b}\right) M^{\prime}+a M\|h(\cdot)-\mathbb{E} h(W)\|\right) \frac{a^{2}}{(p+5)^{2} g_{2}\left(\frac{p+5}{a}\right)}
$$

and $M$ is given by (3.9) and $M^{\prime}$ by (3.10).

The proof, similar to that of the bound of the first derivative, is given in appendix.

\section{About the Stein equation related to the Kummer distribution}

Recall that for $a>0, b \in \mathbb{R}, c>0$, the Kummer distribution $K(a, b, c)$ has density

$$
k_{a, b, c}(x)=\frac{1}{\Gamma(a) \psi(a, a-b+1 ; c)} x^{a-1}(1+x)^{-a-b} e^{-c x},(x>0)
$$

where $\psi$ is the confluent hypergeometric function of the second kind. Let

$$
s(x)=x(1+x) \quad \text { and } \quad \tau(x)=(1-b) x-c x(1+x)+a .
$$

Then

$$
\left(s(x) k_{a, b, c}(x)\right)^{\prime}=\tau(x) k_{a, b, c}(x) .
$$

Then we can use Theorem 2.1 to obtain the following Stein characterization of the Kummer distribution:

Theorem 4.1. A random variable $X$ follows the $K(p, a, b)$ distribution if and only if, for all differentiable functions $f$ such that the expectation exists,

$$
\mathbb{E}\left[X(X+1)\left(f^{\prime}(X)+((1-b) X-c X(1+X)+a) f(X)\right]=0 .\right.
$$

The corresponding Stein equation is

$$
x(x+1) f^{\prime}(x)+[(1-b) x-c x(1+x)+a] f(x)=h(x)-\mathbb{E} h(W)
$$

where $W$ has density $k_{a, b, c}$.

We have

$$
s(x) k_{a, b, c}(x)=\frac{1}{\Gamma(a) \psi(a, a-b+1 ; c)} x^{a}(1+x)^{1-a-b} e^{-c x}, \quad x>0
$$

and we see that $\lim _{x \rightarrow \infty} s(x) k_{a, b, c}(x)=\lim _{x \rightarrow 0} s(x) k_{a, b, c}(x)=0$.

\subsection{Bound of the solution in the case $1-b-c \leq 0$}

Note that if $1-b-c \leq 0$, then the function $\tau$ defined by (4.1) is decreasing on $(0, \infty)$ and its only zero on this interval is

$$
\alpha=\frac{1-b-c+\sqrt{(1-b-c)^{2}+4 a c}}{2 c} .
$$

Then we use again Proposition 2.4 and Proposition 2.5 to obtain the following result: 
Theorem 4.2. The Kummer Stein equation (4.2) has solution

$$
\begin{aligned}
f_{h}(x) & =\frac{1}{s(x) k_{a, b, c}(x)} \int_{0}^{x} k_{a, b, c}(t)[h(t)-\mathbb{E} h(W)] \mathrm{d} t \\
& =\frac{-1}{s(x) k_{a, b, c}(x)} \int_{x}^{+\infty} k_{a, b, c}(t)[h(t)-\mathbb{E} h(W)] \mathrm{d} t
\end{aligned}
$$

where $W \sim K(a, b, c)$.

If $h$ is a bounded continuous function and $1-b-c \leq 0$, then $f_{h}$ defined by (4.4) is the unique bounded solution of (4.2) and

$$
\left\|f_{h}\right\| \leq M\|h(\cdot)-\mathbb{E} h(W)\|
$$

where

$$
M=\max \left(\frac{1}{s(\alpha) k_{a, b, c}(\alpha)} \int_{0}^{\alpha} k_{a, b, c}(t) \mathrm{d} t ; \frac{1}{s(\alpha) k_{a, b, c}(\alpha)} \int_{\alpha}^{+\infty} k_{a, b, c}(t) \mathrm{d} t\right) .
$$

Remark 4.3. By bounding by 1 the integrals in equation (4.5), one obtains

$$
\begin{aligned}
\left\|f_{h}\right\| & \leq \frac{1}{\alpha(\alpha+1) k_{a, b, c}(\alpha)}\|h(\cdot)-\mathbb{E} h(W)\| \\
& =\frac{2 c^{2}}{\left(2 a c+(1-b)\left(1-b-c+\sqrt{(1-b-c)^{2}+4 a c}\right)\right) k_{a, b, c}\left(\frac{1-b-c+\sqrt{(1-b-c)^{2}+4 a c}}{2 c}\right)}\|h(\cdot)-\mathbb{E} h(W)\| .
\end{aligned}
$$

\subsection{Bound of the solution in the case $1-b-c>0$}

Theorem 4.4. Let $h:(0, \infty) \rightarrow \mathbb{R}$ be a continuous, bounded function. If $1-b-c>0$, then the solution $f_{h}$ of the Stein equation (4.2) for the Kummer distribution with parameters $a>0, b \in \mathbb{R}, c>0$, is such that

$$
\left\|f_{h}\right\| \leq K\|h(\cdot)-\mathbb{E} h(W)\|
$$

where

$$
K=\max \left(\frac{1}{a}, \frac{c^{2}}{(1-b)(1-b-c) k_{a, b, c}\left(\frac{1-b-c}{c}\right)}\right) .
$$

Proof. We apply Proposition 2.5.

In the sequel we give the results bounding the first and second derivatives of the solution of the Kummer Stein equation. We do not give the proofs because the arguments are similar to those used for the GIG Stein equation.

\subsection{Bound of the derivative}

Theorem 4.5. Let $h$ be a continuous bounded function such that the derivative $h^{\prime}$ of $h$ is bounded. Consider the solution $f_{h}$ of the Stein equation (4.2) related to the Kummer distribution with parameters a,b,c. 


$$
\begin{aligned}
& \text { Let } \alpha_{1}=\frac{3-b-c+\sqrt{(3-b-c)^{2}+4 c(a+1)}}{2 c} \text {. Then } \\
& \qquad f_{h}^{\prime} \| \leq K^{\prime}=\left\{\begin{array}{l}
\max \left(K_{2}, K_{3}\right) \text { if } 3-b-c \leq 0 \\
\max \left(K_{1}, K_{3}, K_{4}\right) \text { if } 3-b-c>0
\end{array}\right.
\end{aligned}
$$

where

$$
\begin{gathered}
K_{1}=\frac{1}{a+1}\left(\left\|h^{\prime}\right\|+\max (2,|1-b-c|) K\|h(\cdot)-\mathbb{E} h(W)\|\right), \\
K_{2}=\left(\left\|h^{\prime}\right\|+\left(2+\sqrt{(3-b-c)^{2}+4 c(a+1)}\right) K\|h(\cdot)-\mathbb{E} h(W)\|\right) \frac{1}{\alpha_{1}\left(1+\alpha_{1}\right) k_{a+1, b-2, c}\left(\alpha_{1}\right)} \int_{0}^{\alpha_{1}} k_{a+1, b-2, c}(t) \mathrm{d} t \\
K_{3}=\frac{2}{\alpha_{1}\left(1+\alpha_{1}\right)}\|h(\cdot)-\mathbb{E} h(W)\|, \\
K_{4}=\left(\left\|h^{\prime}\right\|+\left(2+\sqrt{(3-b-c)^{2}+4 c(a+1)}\right) K\|h(\cdot)-\mathbb{E} h(W)\|\right) \frac{c^{2}}{(3-b)(3-b-c) k_{a+1, b-2, c}\left(\frac{3-b-c}{c}\right)}
\end{gathered}
$$

and $K$ is given by

$$
K=\left\{\begin{array}{l}
\max \left(\frac{1}{s(\alpha) k_{a, b, c}(\alpha)} \int_{0}^{\alpha} k_{a, b, c}(t) \mathrm{d} t ; \frac{1}{s(\alpha) k_{a, b, c}(\alpha)} \int_{\alpha}^{+\infty} k_{a, b, c}(t) \mathrm{d} t\right) \quad \text { if } 1-b-c \leq 0 \\
\max \left(\frac{1}{a}, \frac{c^{2}}{(1-b)(1-b-c) k_{a, b, c}\left(\frac{1-b-c}{c}\right)}\right) \text { if } 1-b-c>0
\end{array}\right.
$$

\subsection{Bound of the second derivative}

Theorem 4.6. Let $h$ be a bounded, twice differentiable function such that $h^{\prime}$ and $h^{\prime \prime}$ are bounded. Let

$$
\alpha_{2}=\frac{5-b-c+\sqrt{(5-b-c)^{2}+4 c(a+2)}}{2 c} .
$$

The second derivative $f_{h}^{\prime \prime}$ of the solution $f_{h}$ of equation (4.2) is such that

$$
\left\|f_{h}^{\prime \prime}\right\| \leq K^{\prime \prime}=\left\{\begin{array}{l}
\max \left(K_{5}, K_{6}\right) \quad \text { if } 5-b-c \leq 0 \\
\max \left(K_{6}, K_{7}, K_{8}\right) \text { if } 5-b-c>0
\end{array}\right.
$$

where

$$
\begin{aligned}
K_{5}= & \left(\left\|h^{\prime \prime}\right\|+\left(6+2 \sqrt{(5-b-c)^{2}+4 c(a+2)}\right) K^{\prime}+a K\|h(\cdot)-\mathbb{E} h(W)\|\right) \\
& \times \frac{1}{\alpha_{2}\left(\alpha_{2}+1\right) k_{a+2, b-4, c}\left(\alpha_{2}\right)} \int_{0}^{\alpha_{2}} k_{a+2, b-4, c}(t) \mathrm{d} t,
\end{aligned}
$$




$$
\begin{gathered}
K_{6}=\frac{2}{\alpha_{2}\left(1+\alpha_{2}\right)}\left(\left\|h^{\prime}\right\|+\frac{4 c+c \sqrt{(5-b-c)^{2}+4 c(a+2)}}{10-2 b+2 \sqrt{(5-b-c)^{2}+4 c(a+2)}}\|h(\cdot)-\mathbb{E} h(W)\|\right), \\
K_{7}=\frac{1}{a+2}\left(\left\|h^{\prime \prime}\right\|+\max (6,|4-2 b-2 c|) K^{\prime}+2 c K\|h(\cdot)-\mathbb{E} h(W)\|\right), \\
K_{8}=\left(\left\|h^{\prime \prime}\right\|+\left(6+2 \sqrt{(5-b-c)^{2}+4 c(a+2)}\right) K^{\prime}+a K\|h(\cdot)-\mathbb{E} h(W)\|\right) \\
\quad \times \frac{c^{2}}{(5-b)(5-b-c) k_{a+2, b-4, c}\left(\frac{5-b-c}{c}\right)},
\end{gathered}
$$

$K$ is given by (4.7) and $K^{\prime}$ by (4.6).

\section{Potential applichtions}

These results could be used to provide rates of convergence in limit problems related to the GIG and Kummer distributions. Here are two instances of applications that will be considered in future work.

\subsection{The GIG distribution as the law of a continued fraction}

\section{Theorem 5.1. [8]}

- Let $X$ and $Y$ be two independent random variables such that $X>0$ and $Y \sim \gamma(p, a / 2)$ for $p, a>0$, where $\gamma(p, c)$ is the gamma distribution with density proportional to $x^{p-1} e^{-c x}$.

Then $X={ }_{d} \frac{1}{Y+X}$ if and only if $X \sim \operatorname{GIG}(-p, a, a)$.

- Let $X, Y_{1}$ and $Y_{2}$ be three independent random variables such that $X>0, Y_{1} \sim \gamma(p, b / 2)$ and $Y_{2} \sim \gamma(p, a / 2)$ for $p, a, b>0$. Then $X={ }_{d} \frac{1}{Y_{1}+\frac{1}{Y_{2}+X}}$ if and only if $X \sim \mathrm{GIG}(-p, a, b)$.

- If $\left(Y_{i}\right)_{i \geq 1}$ is a sequence of independent random variables such that

$$
\mathcal{L}\left(Y_{2 i-1}\right)=\mathcal{L}\left(Y_{1}\right)=\gamma(\lambda, b / 2) \quad \text { and } \quad \mathcal{L}\left(Y_{2 i}\right)=\mathcal{L}\left(Y_{2}\right)=\gamma(\lambda, a / 2) ; \quad i \geq 1,
$$

then

$$
\mathcal{L}\left(\frac{1}{Y_{1}+\frac{1}{Y_{2}+\frac{1}{Y_{3}+\ddots}}}\right)=G I G(-\lambda, a, b)
$$

A natural continuation of this work would be to derive bounds for the distance between

$$
\mathcal{L}\left(\frac{1}{Y_{1}+\frac{1}{Y_{2}+\frac{1}{\cdots+\frac{1}{Y_{n}}}}}\right)
$$

and $\operatorname{GIG}(p, a, b)$. 


\subsection{The Kummer distribution as the law of a continued fraction}

\section{Theorem 5.2. [5]}

- Let $X, Y_{1}$ and $Y_{2}$ be three independent random variables such that $Y_{1} \sim \gamma(a, c)$ and $Y_{2} \sim \gamma(a+b, c)$ for $a, c>0$ and $b>-a$. Then $X={ }_{d} \frac{Y_{1}}{1+\frac{Y_{2}}{1+X}}$ if and only if $X \sim \mathrm{K}(a, b, c)$.

- If $\left(Y_{i}\right)_{i \geq 1}$ is a sequence of independent random variables such that

$$
\mathcal{L}\left(Y_{2 i-1}\right)=\mathcal{L}\left(Y_{1}\right)=\gamma(a, c) \quad \text { and } \quad \mathcal{L}\left(Y_{2 i}\right)=\mathcal{L}\left(Y_{2}\right)=\gamma(a+b, c) ; \quad i \geq 1,
$$

then

$$
\mathcal{L}\left(\frac{Y_{1}}{1+\frac{Y_{2}}{1+\frac{Y_{3}}{1++\ddots}}}\right)=K(a, b, c)
$$

Finding bounds for the distance between

$$
\mathcal{L}\left(\frac{Y_{1}}{1+\frac{Y_{2}}{1+\frac{Y_{3}}{1+\cdots+\frac{Y_{n-1}}{1+Y_{n}}}}}\right)
$$

and $\mathrm{K}(a, b, c)$ may be another question of interest.

\section{Appendix A. Proof of Theorem 3.7}

First, assume that $p \leq-5$. $f_{h}^{\prime \prime}$ satisfies the differential equation

$$
x^{2} f^{(3)}(x)+\left(\frac{b}{2}+(p+5) x-\frac{a}{2} x^{2}\right) f^{\prime \prime}(x)=h^{\prime \prime}(x)-2(p+2-a x) f^{\prime}(x)+a f(x) .
$$

Let $h_{2}(x)=h^{\prime \prime}(x)-2(p+2-a x) f_{h}^{\prime}(x)+a f_{h}(x)$. Equation (A.1) shows that $f_{h}^{\prime \prime}$ solves the Stein equation for $G I G(p+4, a, b)$ distribution with right-hand side $h_{2}(x)=h^{\prime \prime}(x)-2(p+2-a x) f_{h}^{\prime}(x)+a f_{h}(x)$. Let

$$
s(x)=x^{2} \quad \text { and } \quad \tau_{2}(x)=\frac{b}{2}+(p+5) x-\frac{a}{2} x^{2},
$$

we have

$$
\left(s(x) g_{2}(x)\right)^{\prime}=\tau_{2}(x) g_{2}(x)
$$

where $g_{2}$ is the density of $G I G(p+4, a, b)$ distribution.

For $p \leq-5, \tau_{2}$ is decreasing on $(0, \infty)$ and its only zero is $\alpha_{2}=\frac{p+5+\sqrt{(p+5)^{2}+a b}}{a}$. If $x<\alpha_{2}$, 
Theorem 3.5 shows that, if $h_{2}$ is bounded, the solution $f_{h}^{\prime \prime}$ of equation (A.1) is such that

$$
\left\|f_{h}^{\prime \prime}\right\| \leq\left\|h_{2}\right\| \frac{1}{s\left(\alpha_{2}\right) g_{2}\left(\alpha_{2}\right)} \int_{0}^{\alpha_{2}} g_{2}(t) \mathrm{d} t
$$

We have

$$
\begin{aligned}
\left|h_{2}(x)\right| & =\left|h^{\prime \prime}(x)-2(p+2-a x) f_{h}^{\prime}(x)+a f_{h}(x)\right| \\
& \leq\left\|h^{\prime \prime}\right\|+2|p+2-a x|\left\|f_{h}^{\prime}\right\|+a\left\|f_{h}\right\| \\
& =\left\|h^{\prime \prime}\right\|+2(-p-2+a x)\left\|f_{h}^{\prime}\right\|+a\left\|f_{h}\right\| \quad(p+2-a x<0 \quad \forall p \leq-5, x>0) \\
& \leq\left\|h^{\prime \prime}\right\|+2\left(-p-2+a \alpha_{2}\right)\left\|f_{h}^{\prime}\right\|+a\left\|f_{h}\right\| \quad\left(x<\alpha_{2}\right) \\
& =\left\|h^{\prime \prime}\right\|+\left(6+2 \sqrt{(p+5)^{2}+a b}\right)\left\|f_{h}^{\prime}\right\|+a\left\|f_{h}\right\| \\
& \leq\left\|h^{\prime \prime}\right\|+\left(6+2 \sqrt{(p+5)^{2}+a b}\right) M^{\prime}+a M\|h(\cdot)-\mathbb{E} h(W)\| .
\end{aligned}
$$

Hence for $p \leq-5$ and $x \leq \alpha_{2}$,

$$
\begin{aligned}
\left|f_{h}^{\prime \prime}(x)\right| \leq & \left(\left\|h^{\prime \prime}\right\|+\left(6+2 \sqrt{(p+5)^{2}+a b}\right) M^{\prime}+a M\|h(\cdot)-\mathbb{E} h(W)\|\right) \\
& \times \frac{1}{s\left(\alpha_{2}\right) g_{2}\left(\alpha_{2}\right)} \int_{0}^{\alpha_{2}} g_{2}(t) \mathrm{d} t .
\end{aligned}
$$

If $p \leq-5$ and $x \geq \alpha_{2}$, the function $\tau_{1}$ defined by $\tau_{1}(x)=\frac{b}{2}+(p+3) x-\frac{a}{2} x^{2}$ is decreasing and negative. By equation (3.11),

$$
\begin{aligned}
f_{h}^{\prime \prime}(x) & =\frac{h_{1}(x)}{s(x)}-\frac{\tau_{1}(x) f_{h}^{\prime}(x)}{s(x)} \\
\left|f_{h}^{\prime \prime}(x)\right| & \leq \frac{\left\|h_{1}\right\|}{s(x)}+\frac{\left|\tau_{1}(x) f_{h}^{\prime}(x)\right|}{s(x)} \\
\left|\tau_{1}(x) f_{h}^{\prime}(x)\right| & =\left|\frac{-\tau_{1}(x)}{s(x) g_{1}(x)} \int_{x}^{+\infty} g_{1}(t) h_{1}(t) \mathrm{d} t\right| \\
& \leq\left\|h_{1}\right\| \frac{-\tau_{1}(x)}{s(x) g_{1}(x)} \int_{x}^{+\infty} g_{1}(t) \mathrm{d} t \quad\left(\tau_{1} \quad \text { is negative }\right) \\
& \leq\left\|h_{1}\right\| \frac{-\tau_{1}(x)}{s(x) g_{1}(x)} \int_{x}^{+\infty} \frac{\tau_{1}(t)}{\tau_{1}(x)} g_{1}(t) \mathrm{d} t \quad\left(\frac{\tau_{1}(t)}{\tau_{1}(x)} \geq 1 \quad \forall x \leq t\right) \\
& =\left\|h_{1}\right\| . \\
h_{1}(x)=h^{\prime}(x) & -(p+1-a x) f_{h}(x) \Longrightarrow\left|h_{1}(x)\right| \leq\left\|h^{\prime}\right\|+\left|(p+1-a x) f_{h}(x)\right| .
\end{aligned}
$$

If $p \leq-5$ and $x \geq \alpha_{2}$, the functions $\tau:=\tau_{p, a, b}$ (given by (3.2)) and $x \mapsto p+1-a x$ are decreasing and negative. Then

$$
\begin{aligned}
\left|(p+1-a x) f_{h}(x)\right| & =\left|-\frac{p+1-a x}{s(x) g_{p, a, b}(x)} \int_{x}^{+\infty} g_{p, a, b}(t)[h(t)-\mathbb{E} h(W)] \mathrm{d} t\right| \\
& \leq\|h(\cdot)-\mathbb{E} h(W)\| \frac{-p-1+a x}{s(x) g_{p, a, b}(x) \tau(x)} \int_{x}^{+\infty} \tau(t) g_{p, a, b}(t) \mathrm{d} t \quad\left(\frac{\tau(t)}{\tau(x)} \geq 1 \quad \forall x \leq t\right) \\
& =\|h(\cdot)-\mathbb{E} h(W)\| \frac{p+1-a x}{\tau(x)} .
\end{aligned}
$$


Let $\kappa(x)=\frac{p+1-a x}{\tau(x)} . \kappa$ is differentiable on $\left(\alpha_{2}, \infty\right)$ and $\kappa^{\prime}(x)=-\frac{a b+(p+1)^{2}+(p+1-a x)^{2}}{2 \tau^{2}(x)} \leq 0$. Hence for $p \leq-5$ and $x \geq \alpha_{2}, \kappa(x) \leq \kappa\left(\alpha_{2}\right)=\frac{p+1-a \alpha_{2}}{\tau\left(\alpha_{2}\right)}=\frac{4 a+a \sqrt{(p+5)^{2}+a b}}{4(p+5)+4 \sqrt{(p+5)^{2}+a b}}$ and

$$
\left\|f_{h}^{\prime \prime}\right\| \leq \frac{2}{\alpha_{2}^{2}}\left(\left\|h^{\prime}\right\|+\frac{4 a+a \sqrt{(p+5)^{2}+a b}}{4 p+20+4 \sqrt{(p+5)^{2}+a b}}\|h(\cdot)-\mathbb{E} h(W)\|\right) .
$$

Hence $\left\|f_{h}^{\prime \prime}\right\| \leq \frac{2}{\alpha_{2}^{2}}\left(\left\|h^{\prime}\right\|+\left(4+\sqrt{(p+5)^{2}+a b}\right) M\|h(\cdot)-\mathbb{E} h(W)\|\right)$.

Now assume that $p>-5$.

On $\left(0, \frac{p+5}{a}\right), \tau_{2}$ is such that $\frac{\tau_{2}(t)}{\tau_{2}(0)} \geq 1 \forall 0 \leq t \leq \frac{p+5}{a}$, so that

$$
\begin{aligned}
\left|h_{2}(x)\right| & \leq\left\|h^{\prime \prime}\right\|+2|p+2-a x|\left\|f_{h}^{\prime}\right\|+a\left\|f_{h}\right\| \\
& \leq\left\|h^{\prime \prime}\right\|+\max (6,2 p+4)\left\|f_{h}^{\prime}\right\|+a\left\|f_{h}\right\| \\
& \leq\left\|h^{\prime \prime}\right\|+\max (6,2 p+4) M^{\prime}+a M\|h(\cdot)-\mathbb{E} h(W)\| .
\end{aligned}
$$

As a consequence,

$$
\begin{aligned}
\left|f_{h}^{\prime \prime}(x)\right| & \leq\left\|h_{2}\right\| \frac{1}{s(x) g_{2}(x)} \int_{0}^{x} g_{2}(t) \mathrm{d} t \\
& \leq\left\|h_{2}\right\| \frac{1}{\tau_{2}(0) s(x) g_{2}(x)} \int_{0}^{x} \tau_{2}(t) g_{2}(t) \mathrm{d} t \\
& =\frac{1}{\tau_{2}(0)}\left\|h_{2}\right\| \\
& =\frac{2}{b}\left\|h_{2}\right\|
\end{aligned}
$$

and

$$
\left\|f_{h}^{\prime \prime}\right\| \leq \frac{2}{b}\left(\left\|h^{\prime \prime}\right\|+\max (6,2 p+4) M^{\prime}+a M\|h(\cdot)-\mathbb{E} h(W)\|\right) .
$$

On $\left(\frac{p+5}{a}, \infty\right)$, the function $\tau_{2}$ is decreasing and similar arguments as in the case $p \leq-5$ lead to the result.

Acknowledgements. We are grateful to a referee whose remarks improved the content of this paper.

\section{REFERENCES}

[1] L.H.Y. Chen, L. Goldstein and Q.-M. Shao, Normal approximation by Stein's method. Probability and its Applications Heidelberg (2011).

[2] R.E. Gaunt, A Stein characterization of the generalized hyperbolic distribution. ESAIM: PS 21 (2017) $303-316$.

[3] R.E. Gaunt, A.M. Pickett and G. Reinert, Chi-square approximation by Stein's method with application to Pearson's statistic. Ann. Appl. Probab. 27 (2017) 720-756.

[4] L. Goldstein and G. Reinert, Stein's method for the Beta distribution and the Pòlya-Eggenberger urn. Adv. Appl. Probab. 50 (2013) 1187-1205.

[5] M. Hamza and P. Vallois, On Kummer's distribution of type two and a generalized beta distribution. Statist. Prob. Lett. 118 (2016) 60-69.

[6] A.E. Koudou and P. Vallois, Independence properties of the Matsumoto-Yor type. Bernoulli 18 (2012) $119-136$. 
[7] A.E. Koudou and C. Ley, Characterizations of GIG laws: a survey complemented with two new results. Probab. Surv. 11 (2014) 161-176.

[8] G. Letac and V. Seshadri, A characterization of the generalized inverse Gaussian distribution by continued fractions. $Z$. Wahr. verw. Geb. 62 (1983) 485-489.

[9] C. Ley and Y. Swan, Stein's density approach and information inequalities. Electron. Comm. Probab. 18 (2013) 1-14.

[10] A. Piliszek and J. Wesołowski, Change of measure technique in characterizations of the gamma and Kummer distributions. $J$. Math. Anal. Appl. 458 (2018) 967-979.

[11] N. Ross, Fundamentals of Stein's method. Probab. Surv. 8 (2011) 210-293.

[12] W. Schoutens, Orthogonal polynomials in steins method. J. Math. Anal. Appl. 253 (2001) 515-531.

[13] Q.-M. Shao and Z.-S. Zhang, Identifying the limiting distribution by a general approach of Stein's method. Sci. China Math. 59 (2016) 2379-2392.

[14] C. Stein, A bound for the error in the normal approximation to the distribution of a sum of dependent random variables, in Vol. 2 of Proceedings of the Sixth Berkeley Symposium on Mathematical Statistics and Probability. University of California Press, Berkeley (1972) 583-602.

[15] C. Stein, P. Diaconis, S. Holmes and G. Reinert, Use of exchangeable pairs in the analysis of simulations, in Stein's method: expository lectures and applications, edited by Persi Diaconis and Susan Holmes. Vol. 46 of IMS Lecture Notes Monogr. Ser. Institute of Mathematical Statistics, Beachwood, Ohio, USA (2004) 1-26. 